\title{
Hommage à Maximilien Laroche
}

\section{Homage to Maximilien Laroche}

Nubia Hanciau ${ }^{1}$

Zilá Bernd ${ }^{2}$

Submetido em 4 de agosto e aprovado em 8 de agosto de 2017.

En 2010, Nubia Hanciau, éditrice à l'époque de la revue Interfaces Brasil-Canadá a eu la bonne idée d'interpréter la pensée de ses collègues brésiliens - qui admiraient profondément Maximilien Laroche - de lui rendre hommage. Elle a très bien souligné les principales caractéristiques de cet intellectuel qui, vivant au Québec et enseignant à 1'Université Laval, était né en Haïti, pays qui revient constamment dans ses réflexions. Une magnifique idée celle de rendre hommage à ce collègue qui, à part d'être un éminent professeur et penseur, était aussi un être humain incomparable par son immense capacité de s'ouvrir à la relation avec les autres dans la diversité.

L'idée de notre collègue Nubia était parfaite car Maximilien Laroche a eu le temps de lire et de savoir tout le respect et toute l'admiration que ses lecteurs au Brésil avaient à son égard. Maintenant que l'on doit annoncer la triste nouvelle de son décès le 27 juillet dernier, nous pensons à traduire son texte vers le français pour que les amis du Québec et surtout pour que sa famille puissent lire ce témoignage si profondément sincère et véritable.

«L'hommage que nous rendons ici permet de parcourir la trajectoire et revoir à travers la mémoire le chemin des mots qui ont séduit et orienté toute une génération de collègues québécistes et canadianistes, amis et fidèles qui suivaient les réflexions de Maximilien Laroche. Prévu pour se dérouler pendant le X Congrès Brésilien d'Études Canadiennes, à Goiânia, en 2009 - quand une table-ronde avec sa participation était organisée -, «Maxi», comme affectueusement Maximilien Laroche était appelé, pour des raisons qui échappent à notre contrôle, n'a pas pu se faire présent. Nous essaierons ici de combler cette lacune symboliquement, en ouvrant la présente édition du n. 11 de 2010, de la revue Interfaces Brasil-Canada, avec un de ses textes, véritable primeur du comparatisme littéraire qu'il a toujours exercé dans un niveau d'excellence. Maxi est 
ainsi vivement présent dans notre remerciement par son travail pionnier de défrichage de la route Nord-Sud des études canadiennes et québécoises et par son rôle fondamental aux échanges entre nos pays depuis le début des années 1980. Si les mots volent, l'écriture reste. Dans cette édition ses mots persisteront ainsi que notre reconnaissance. Les missions académiques annuelles de Maximilien Laroche au Brésil se sont déroulées en alternance aux universités Fédérales Fluminense, du Rio Grande do Sul, du Paraná, ainsi qu'à l'Université de São Paulo, Universités d'état de Bahia (UNEB e UEFS) et Paulista Julio de Mesquita Filho.

Au-delà de l'intérêt pour les écrivains qui sont originaires d'Haïti, du Québec et du Canada, son regard se tourne vers les auteurs et poètes brésiliens, en préfigurant des espaces transnationaux dans le cadre du mouvement général des peuples, dont la pertinence est aussi grande aujourd hui qu'à l'occasion de la publication de son livre Dialectique de l'Américanisation (1993). Dans cet ouvrage, qui se démarque parmi les nombreux titres que nous avons l'habitude de consulter et de citer dans nos recherches, M. Laroche nous dit qu'être «américain», depuis 500 ans d'une Histoire qui adhère à la peau comme un vêtement étroit, correspond à rêver d'une Histoire à venir.

La métamorphose que cette terre et son histoire imposent est vécue par le comparatiste qui l'articule à plusieurs théories, transformées en instrumental théorique et méthodologique pour les analyses des questions interlittéraires, interdiscursives et interdisciplinaires, dans les champs les plus divers de la recherche littéraire et culturelle. Ce simple hommage des canadianistes brésiliens et de la revue Interfaces Brasil/Canadá à Maximilien Laroche suit d'autres plus importants, tels que le Doctorat Honoris Causa lequel lui a été attibué par McMaster University, en 2009, le titre de Chevalier de l'Ordre de 1'Honneur et Mérite de la République d'Haïti, la Médaille du Conseil Général de la Martinique, la Médaille d'Argent de la Société des Amis d'Alexandre Dumas, l'Ordre des Francophones des Amériques. Poète, inventif, érudit, philosophe, maître d'un imaginaire savoureux et d'une langue richissime, s'exprimant tant en français comme en créole, auteur des récits qui se démarquent par leur originalité thématique, par son «américanité», par son style et ton particuliers, professeur et écrivain, cap-haïtien de naissance, devenu citoyen canadien, Maximilien Laroche s'inscrit dans la catégorie des 
Joël Des Rosiers, Émile Ollivier, Aimé Césaire, Édouard Glissant, Derek Walcot, Gérard Étienne, Neil Bissoondath, V. S. Naipaul, Max Dorsinville, et des nombreux poètes et romanciers, critiques latino-americains.

$\mathrm{C}^{\prime}$ est bien dans les profondeurs de la langue, de la littérature, du réalisme merveilleux, de la mémoire des lieux que plonge l'écrivain Maximilien Laroche, lui qui repense l'écriture en tant que science ».

Encore sous le choc de la triste nouvelle du passement de notre collègue et ami, mais réconfortés du fait de savoir qu'il est mort dans les bras de sa chère épouse Xin Du et de ses deux enfants, Catherine et Olivier Laroche, nous voulons signaler une fois de plus l'importance pour nos carrières de professeurs de français en Amérique latine, de comprendre une des leçons majeures de M. Laroche: l'importance de mieux comprendre la culture des Amériques et d'établir un dialogue inter et trans-américain à travers la langue française.

Dans un de ses derniers livres intitulé Le poids des mots (Québec, 2013), cet homme pour qui les mots «étaient tout», nous livre une leçon inoubliable:

Nous mesurons mal ce poids des mots dont nous nous servons. Il ne nous apparaît qu'au fil du temps. Il nous faut donc sans cesse sonder ce poids pour voir comment il se réincarne dans le réel que nous essayons d'éclairer.

Les collègues, les amis et tous ceux qui s'intéressent aux littératures francophones de la Caraïbe, du Québec, d'Afrique ou de l'Océan Indien, ainsi que tous ceux qui travaillent pour déchiffrer les relations littéraires entre les «Amériques», se sentent en ce moment orphelins et orphelines, comme nous a écrit Jean Claude Castelain, collègue montréalais originaire de l'île Maurice et grand admirateur de M. Laroche:

Nous voilà orphelines et orphelins de notre merveilleux grand frère Maxi ce soir. Comme je l'écrivais à Vinesh (Hookoonsing), c'est une très triste et terrible nouvelle, une immense perte pour nous tous. Notre poto-mitan s'en est allé et laisse un grand vide.

Quand son épouse Xin m'a annoncé cela ce soir au téléphone, j’ai subi un terrible choc et je suis totalement désemparé. La mince satisfaction est que Maxi est parti sans trop souffrir. J'étais encore récemment en contact avec lui (je lui avais envoyé des articles sur le nouveau doyen linguiste-créoliste de la Faculté des lettres 
de l'université de Maurice ainsi que sur la romancière brésilienne Conceição Evaristo) et comptais aller le voir bientôt. L'écho de son rire résonnera toujours parmi nous de même que je ne vais jamais oublier son 'Mon chè Jan-Klod' à mon égard, quand nous discutions régulièrement au téléphone de la littérature de nos îles, du monde créole, de l'esclavage, de métissage, de rhum, de séga et autres musiques, de recettes culinaires épicées, de politique à Maurice ou en Haïti, de tout et de rien. Le tout assaisonné d'un grand éclat de rire!

Il va me et nous manquer sérieusement, ce grand frère parti dorénavant rejoindre les Ancêtres. Nous devrions tous boire notre meilleur rhum à sa mémoire en écoutant un séga, un konpa, un zouk ou une samba sans oublier de faire une libation (au rhum Barbancourt de préférence) à sa mémoire afin de respecter la tradition. 'Oner \& respe' très cher Maxi, Magnifico Professor Laroche (comme j'avais l'habitude de l'interpeller)!

Jean Claude Castelain conclue son message avec des mots en créole en hommage au grand intérêt que portait Maximilien à l'étude du créole haïtien, langue parlée en Haïti à côté du français et qui a une expression littéraire assez importante que Maximilien Laroche a étudié et publié sous forme d'anthologies. Sa contribution à l'étude des mythes haïtiens et caraïbéens est aussi considérable et on peut la conférer dans l'ouvrage qu'il a publié en 2002 intitulé: Mythologie Haïtienne (Université Laval, v. 18 de la collection GRELCA qu'il dirigeait). Il a également contribué au Dictionnaire des figures et mythes littéraires des Amériques (BERND, 2007), en composant un article sur la figure mythique haïtienne du Bizango qui, à exemple de certaines figures mythologiques des Amériques, est synonyme de transformation, métamorphose, comme le zombi et le loup-garou. Nous croyons ici au sud de 1'Equateur que 1'esprit de Maximilien Laroche, tel les figures mythiques d'Haiti, continuera à nous illuminer à travers les remarquables ouvrages qu'il nous a laissés.

Nous reprendrons pour terminer, les mots que Suzanne Crosta, professeur à McMaster University (Canada) et grande amie de M. Laroche, a prononcés lors de funérailles de 1'écrivain:

En Maxi, nous perdons un penseur infatigable, un père et époux dévoué, un ami fidèle et enthousiaste, un esprit rieur. Un géant de la littérature haïtienne s'est éteint et il nous manquera profondément.

Août, 2017. 


\section{Références}

BERND, Z. (Org.). Dicionário de figuras e mitos literários das Américas. Porto Alegre: Tomo Editorial; Ed. da UFRGS, 2007.

HANCIAU, Nubia. Homenagem a Maximilien Laroche. Interfaces Brasil/Canadá, Rio Grande, v. 10, n. 1, p. 15-16, 2010.

LAROCHE, Maximilien. Dialectique de l'Américanisation. Quebec: Université Laval/ GRELCA, 1993. . Mythologie Haïtienne. Quebec: Université Laval/GRELCA, 2002. . Bref commentaire sur trois poèmes d'Oswald Durand et un récit de Dany Laferrière.

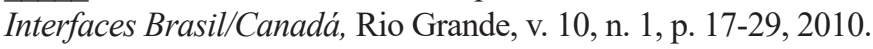
. Le poids des mots. Québec: GRELCA, 2013.

\section{Notes}

${ }^{1}$ Professora Aposentada da FURG, Rio Grande, RS. Colaboradora no Programa de Pós-Graduação em Letras, História da Literatura.nubiajh@gmail.com

2 Professora do PPG-Memória Social e Bens Culturais da Universidade LaSalle, Canoas, Rio Grande do Sul. Pesquisadora 1b do CNPQ. zilabster@gmail.com 